\title{
Unobtrusive Human Localization and Activity Recognition for Supporting Independent Living of the Elderly
}

\author{
Wenjie Ruan \\ School of Computer Science, The University of Adelaide, SA 5005, Australia \\ Email:wenjie.ruan@adelaide.edu.au;
}

\begin{abstract}
Indoor localization and activity recognition is a fundamental research topic for a wide range of important applications such as fall detection of elderly people. It usually requires an intelligent environment to successfully infer where and what a person is doing. However, many of the existing techniques on localization and activity recognition rely heavily on people's involvement such as wearing battery-powered sensors, which might not be practical in real-world situations (e.g., people may forget to wear sensors). In this project, we propose a device-free localization and activity recognition approach using passive RFID tags. It is achieved by learning how the Received Signal Strength Indicator (RSSI) from the passive RFID tag array is distributed when a person performs different activities in different locations. After activity patterns are discovered for a particular individual, we will also develop a context-aware, common-sense based activity reasoning engine that assists applications to make appropriate interpretation of detected activities. We believe the proposed system has the potential to better support the independent living of elderly people considering the continuously increased aging population.
\end{abstract}

\section{INTRODUCTION}

With recent developments in cheap sensor and networking technologies, it has become possible to develop a wide range of valuable applications such as the remote health monitoring and intervention [1]. These applications offer the potential to enhance the quality of life for the elderly, afford them a greater sense of security, and facilitate independent living [2]. Central to realizing these applications is to study low cost sensor technologies that are practical for human localization and activity recognition, particularly for older people [3]. However, existing approaches either rely on body-worn sensors to detect human's location/activity [4], or dense sensing where low cost sensors (e.g., wireless transceivers) are attached to objects and peoples activities can be indirectly inferred from their interactions with the objects [5], [6]. In the former approaches, battery powered sensors are normally bigger in size, expensive, and require maintenance and user involvement (e.g., wearing the device). In the latter approaches, sensors are typically cheaper and maintenance free, however, user involvement is still needed (e.g., wearing a bracelet to detect objects). All these technologies are not very practical, especially for monitoring aged people with dementia. In addition, they can only recognise pre-selected activities and do not deal with behavior changes (e.g., even same individual might perform a same activity in different ways) [7].
Recent advancement in low-cost passive Radio-Frequency Identification (RFID) tags makes device-free (or unobtrusive) activity recognition possible [8], [9]. They are maintenance free (no batteries in tags) and inexpensive (about 5 20 cents each and still dropping quickly). As a result, this project proposes to develop a novel system for automated location monitoring and human activity discovery based on unobtrusive RFID technology. In the first stage, we will design a devicefree system that can continuously track a resident's daily walking route and real-time recognizes human activity using an array of pure passive RFID tags [10], [8].

However, without considering context information (e.g., time and people's age) and common-sense knowledge, activities and locations detected from previous stage present little value. For example, for the activity of LyingDown, LyingDown in bedroom and LyingDown in kitchen may have very different meanings. The latter may indicate an accident that needs urgent attention. The final goal of this task is therefore to develop a context-aware, common-sense based activity reasoning engine that assistants applications to make appropriate interpretation of detected activities and locations. In this project, we propose to build an ontology based on OpenCyc [11] - the world's largest and most complete commonsense knowledge base that contains more than 300,000 concepts - for activity reasoning. Overall, we believe the proposed project can help the elderly live longer independently and safely in their own homes, with minimal support from the decreasing number of individuals in the working-age population. In a nutshell, our main contributions are summarized as follows:

- We introduce a pure passive tag-based localization and tracking system. To the best of our knowledge, our work is one of very first to deal with device-free localization and tracking based on pure passive RFID tags.

- We address the activity recognition problem using an array of pure passive RFID tags. Our approach is light-weight, low-cost in the sense that only passive RFID tags are used. Our proposed approach also relaxes the requirement that people need to wear sensors for poster recognition.

- We will design a context-aware, common-sense based activity reasoning engine that assistants applications to make appropriate interpretation of detected activities 
The rest of the paper is organized as follows. We first discuss the related work in Section II. Then we highlight the proposed system in Section III. Finally, some concluding remarks are offered in Section IV.

\section{RELATED WORK}

A crucial prerequisite of supporting independent living is to accurately localize and track people in an indoor environment [6]. Thus, device-free (or unobstructive) indoor localization systems have been proposed recently [12], [10]. One popular device-free technique category is based on computer vision, such as using RGB camera, depth camera, or infrared sensors [13]. However they are usually regarded as being privacy invasive and cause uncomfortable feeling to the residents. Vision-based systems also require the tracked target with in the line of sight (LOS) of cameras (i.e., no barriers or blocks between the subject and camera), and fail to work in dimmed or dark environments [4]. Another technique category is based on Radio Frequency (RF) signals, e.g., detecting human locations based on Received Signal Strength (RSS), or Channel State Information (CSI) in Wireless Local Area Network (WLAN) [6], or tracking a subject through a wall based on the reflected radio-waves from human body [12]. However, these systems often require specialized WIFI signals or special-purpose devices and regular maintenance (e.g., repalce the batteries regularly).

Another central task of our project is to detect human physical activities from data collected through various kinds of sensors. Computer vision related human activity recognition is one of directions, well-known for its scalability and robustness, but unfortunately, such solutions demand high computational cost for machine interpretation [14]. In addition, the performance of such vision-based approaches depends strongly on the lighting conditions, camera facing angles etc, which greatly restricts its applicability in ubiquitous environments [8]. Also, cameras are generally considered to be intrusive to people's privacy, which makes it hard to widely used in some private environments like home or hospital as not many people enjoy being watched all the time [5]. With the growing maturity of sensor, RFID, and wireless sensor network technologies, activity recognition from inertial, unobtrusive sensor readings has become a popular research area in last few years. Inertial sensors are the most frequently used wearable sensors for human activity recognition [14]. Although sensor-based activity recognition can better address issues such as privacy than conventional computer vision-based approaches, most work from sensor-based activity recognition require people to wear inertial sensors and RFID tags/readers [2]. They have two main shortcomings, one is not convenient to do it, another obstacle is that some people do not want to be equipped with some devices. Therefore these approaches are not always practical, particularly for monitoring elderly persons with cognitive disabilities.

As a result, device-free localization and activity recognition based on passive RFID tags has some promising characters such as low-cost (5 10 cents each, still dropping quickly)

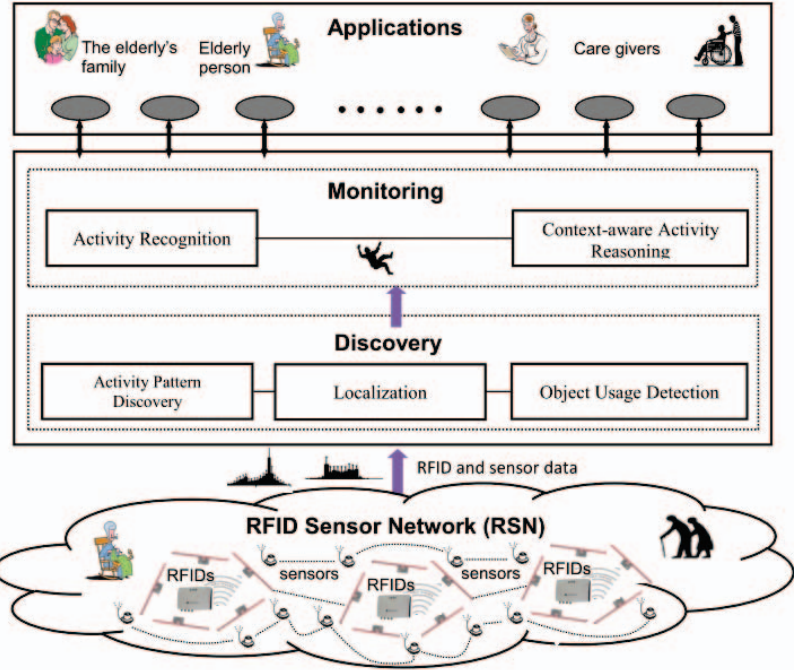

Fig. 1: The overall conceptual framework of the proposed system

and maintenance-free (no batteries needed) [10]. In particular, this project aims to design a system that passively localizes, detects a subject's location and activity to high accuracy with commercial off-the-shelf passive RFID tags.

\section{The Proposed System}

An overview of the overall conceptual framework of our project is depicted in Fig. 1, including three main modules: the Wireless RFID Sensor Network (WRSN), the Activity Discovery (AD), and the Activity Monitoring (AM). The WRSN module investigates the applicability of RFIDs and wireless sensors for human location detection and activity recognition, as well as the deployment of RFID-based sensor networks in laboratory and real-world environments. The AD module automatically discovers the routine activities of individuals using a novel unsupervised approach by mining and clustering RFID and sensor data collected from the WRSN. Finally, the AM module tracks these activities and performs contextaware, common-sense based activity reasoning that will be used by applications (e.g., aged care monitoring systems). To successfully achieve the aims of the project, we carefully divide it into three main tasks:

- Developing a novel device-free localization and tracking system based on the cost-efficient RFID passive tags.

- Developing a device-free, real-time human activity recognition system using an array of passive RFID tags.

- Designing a context-aware, common-sense based activity reasoning engine.

Device-free Indoor Localization: Device-free passive localization aims to localize residents without requiring them to carry any devices or being actively involved with the localizing process, which is a crucial prerequisite of our project [10]. Specifically, we deploy a set of passive RFID tags and a reader (with antennas) formed as an RSS field, enabling the RSSI signal to cover the whole surveillance area. For localizing a 
stationary person, RSSI vectors collected from various known locations along with corresponding correct location labels are used to train classifiers, which are then used to estimate the subject's location given a new RSSI vector. As for the tracking problem, we first augment the traditional $k \mathrm{NN}$ with probabilistic information to model the likelihood of locations based on observed RSSIs, which are used to construct the Emission Matrix in Hidden Markov Model (HMM). Then we estimate the Transmission Matrix under a commonsense-based transferring constraint. At last, we can use Viterbi Search to find the most likely path of the subject, achieving the devicefree tracking.

Unobtrusive Human Activity Recognition: To unobtrusively detect the resident's activity, we deploy passive RFID tags in the residential environment (e.g., on the wall in a bedroom) forming a tag array [8], [5]. We apply the basic principle of sparse representation to realize a robust activity recognition. Specifically, we develop a dictionary-based learning approach to uncover structural information between RSSI signals of different activities. The dictionaries are learned by an unsupervised sparse coding algorithm. In the meantime, we use a lightweight but effective feature selection method to extract signal patterns. We particularly exploit an unsupervised and filter-based feature selection approach based on canonical correlation analysis, which not only retains the natural assignment of feature components but also uncovers the interdependency between feature components. Compared to the existing activity recognition approaches, the proposed dictionary-based approach achieves more compact representation of the activities while preserving richer information, thereby underpinning an efficient and robust recognition of human activities. It should be noted that our approach is scalable in the sense that we learn dictionaries for individual activities so that new activities can be easily accommodated.

Context-aware Activity Reasoning Engine: In this phase, we want to automatically track and monitor each activity and determine if an individual's routine is being maintained and provide timely assistance when needed [2]. We will approach this problem by using a Hidden Markov Model, which is a generative probabilistic model used to generate "hidden" states (e.g., activities) from "evidence" states that can be directly observed (e.g., RFID and sensor readings). The HMM will be trained using the output of our activity pattern discovery algorithm. Activities detected from HMM present little value without considering context information (e.g., time and location) and common-sense knowledge (e.g., at a local time of $3 \mathrm{am}$, sleeping is more likely than walking). Therefore, we will also develop a context-aware, common-sense based activity reasoning engine that assistants applications to make appropriate interpretation of detected activities. One important part of the reasoning engine involves the development of techniques for context information modeling and provisioning. In this project, we will exploit OpenCyc, the world's largest and most complete common-sense knowledge base that contains more than 300,000 concepts. Finally, our work will be to build an ontology based on OpenCyc for activity reasoning.

\section{Conclusion}

My $\mathrm{PhD}$ research aims to automatically discover, identify, and track an individual's routine activities in real-time without any manual annotation of activity data. We have explored low-cost, unobtrusive passive RFID tags for device-free localization and human activity recognition. In the future, we will develop a context-aware, common-sense based activity reasoning engine, and validate the proposed techniques in field trial environments.

\section{ACKNOWLEDGMENT}

Many thanks to my advisors Prof. Michael Sheng, Prof. Xue Li, Dr. Nickolas Falkner and Dr. Lina Yao for their supports and valuable comments. This work has been funded by the Australian Research Council Discovery Project (ARC DP130104614).

\section{REFERENCES}

[1] "2014 state of the smart home, http://www.icontrol.com/blog/2014-statesmart-home/," 2014

[2] W. Ruan, L. Yao, Q. Z. Sheng, N. Falkner, X. Li, and T. Gu, "Tagfall: Towards unobstructive fine-grained fall detection based on uhf passive rfid tags," in Proceedings of International Conference on Mobile and Ubiquitous Systems: Computing, Networking and Services (MobiQuitous'15), 2015.

[3] L. Yao, W. Ruan, Q. Z. Sheng, X. Li, and N. Falkner, "Exploring tagfree rfid-based passive localization and tracking via learning-based probabilistic approaches." in Proceedings of ACM International Conference on Information and Knowledge Management (CIKM 2014), 2014.

[4] Z. Yang, C. Wu, Z. Zhou et al., "Mobility increases localizability: A survey on wireless indoor localization using inertial sensors," ACM Computing Survey, vol. 47, no. 3, pp. 54:1-54:34, 2015.

[5] L. Yao, Q. Z. Sheng, W. Ruan, T. Gu, X. Li, and N. Falkner, "Rf-care: Device-free posture recognition for elderly people using a passive rfid tag array," in Proceedings of International Conference on Mobile and Ubiquitous Systems: Computing, Networking and Services (MobiQuitous 2015), 2015.

[6] Z. Yang, Z. Zhou, and Y. Liu, "From rssi to csi: Indoor localization via channel response," ACM Computing Survey, vol. 46, no. 2, pp. 25:1$25: 32,2013$.

[7] O. Lara and M. Labrador, "A survey on human activity recognition using wearable sensors," Communications Surveys Tutorials, IEEE, vol. 15, no. 3, pp. 1192-1209, 2013.

[8] L. Yao, Q. Z. Sheng, X. Li, S. Wang, T. Gu, W. Ruan, and W. Zou, "Freedom: Online activity recognition via dictionary-based sparse representation of rfid sensing data," in Proceedings of IEEE International Conference on Data Mining (ICDM 2015), 2015.

[9] L. Yao, Q. Z. Sheng, W. Ruan, X. Li, S. Wang, and Z. Yang, "Devicefree posture recognition via online learning of multi-dimensional rfid received signal strength," in Proceedings of the 21th IEEE International Conference on Parallel and Distributed Systems (ICPADS 2015), 2015.

[10] W. Ruan, L. Yao, Q. Z. Sheng, X. Li, and N. Falkner, "Tagtrack: Devicefree localization and tracking using passive rfid tags," in Proceedings of International Conference on Mobile and Ubiquitous Systems: Computing, Networking and Services (MobiQuitous 2014), 2014.

[11] D. B. Lenat, "Cyc: A large-scale investment in knowledge infrastructure," Communications of the ACM, vol. 38, no. 11, pp. 33-38, 1995.

[12] F. Adib, Z. Kabelac, and D. Katabi, "Multi-person localization via rf body reflections," in the 13th USENIX Symposium on Networked Systems Design and Implementation (NSDI 2015), 2015, pp. 279-292.

[13] H. Zhou and H. Hu, "Human motion tracking for rehabilitationa survey," Biomedical Signal Processing and Control, vol. 3, no. 1, pp. 1 - 18, 2008.

[14] S. Mennicken, J. Vermeulen et al., "From today's augmented houses to tomorrow's smart homes: new directions for home automation research," in Proceedings of ACM International Joint Conference on Pervasive and Ubiquitous Computing (UbiComp 2014), 2014. 